\title{
Optimal Rate of Convergence for a Nonstandard Finite Difference Galerkin Method Applied to Wave Equation Problems
}

\author{
Pius W. M. Chin \\ Department of Mathematics and Applied Mathematics, University of Pretoria, Pretoria 0002, South Africa \\ Correspondence should be addressed to Pius W. M. Chin; pius.chin@up.ac.za
}

Received 6 August 2013; Revised 11 November 2013; Accepted 14 November 2013

Academic Editor: Song Cen

Copyright @ 2013 Pius W. M. Chin. This is an open access article distributed under the Creative Commons Attribution License, which permits unrestricted use, distribution, and reproduction in any medium, provided the original work is properly cited.

The optimal rate of convergence of the wave equation in both the energy and the $L^{2}$-norms using continuous Galerkin method is well known. We exploit this technique and design a fully discrete scheme consisting of coupling the nonstandard finite difference method in the time and the continuous Galerkin method in the space variables. We show that, for sufficiently smooth solution, the maximal error in the $L^{2}$-norm possesses the optimal rate of convergence $O\left(h^{2}+(\Delta t)^{2}\right)$ where $h$ is the mesh size and $\Delta t$ is the time step size. Furthermore, we show that this scheme replicates the properties of the exact solution of the wave equation. Some numerical experiments should be performed to support our theoretical analysis.

\section{Introduction}

Most physical phenomena such as the acoustics, electromagnetic, and elastic problems are modeled by the wave equation. The qualitative solution of the model in the spacetime domain is always a very delicate but a fundamental issue that needs careful study. Our point of departure of this paper is to consider the following model of the wave equation: find $u(x, t)$ such that

$$
\begin{gathered}
\frac{\partial^{2} u}{\partial t^{2}}-\Delta u=f \quad \text { in }(x, t) \in \Omega \times(0, T), \\
u(x, 0)=u_{0}(x), \\
\frac{\partial u(x, 0)}{\partial t}=u_{1}(x) \quad x \in \Omega,
\end{gathered}
$$

where $\Omega$ is a smooth bounded domain in $\mathbb{R}^{2}$ with smooth boundary $\partial \Omega$. Problem (1)-(3) consists of constant coefficients and $f(x, t)$ the source term, $u_{0}(x)$ and $u_{1}(x)$ are prescribed as the initial data. Furthermore, $(0, T)$ is taken to be a finite time interval and the boundary conditions satisfied by $u(x, t)$ are given by

$$
u(x, t)=0, \quad(x, t) \in \partial \Omega \times(0, T) .
$$

The methods which have been heavily used for the study of the wave equation (1)-(4) in physical life are the continuous as well as the discontinuous Galerkin methods; see $[1,2]$ for more details. The reason for these methods may be due to the way they deal with heterogeneous media and arbitrary shaped geometric objects, represented by unstructured grids.

The advantages of the continuous Galerkin method are enormous. Firstly, the convergence theory of this method is based on lower regularity (differentiability) requirements than the finite difference and the spectral methods. Secondly, the method retains the important energy conservation properties provided by the discrete version of the initial/boundary valued problem such as the one under consideration. Thirdly, the computation and the analysis from the Galerkin method could be extended in the approximation of the nonlinear wave equation. Furthermore, the method could be applicable to problems of any desired order of accuracy. For more details on these advantages, see in [3-5].

The apriori error estimate for continuous Galerkin approximation of the wave equation (1)-(4) was first derived by Dupont [6] and later improved by Baker [7], both for continuous and discrete time schemes. Gekeler [8] analyzed general multistep methods for the time discretization of secondorder hyperbolic equation, when a Galerkin procedure is 
used in space. The nonclassical finite element treatment of the wave equation can be seen in Johnson [9] and Richter [10].

In this paper, we exploit and present a reliable technique consisting of coupling the nonstandard finite difference (NSFD) method in time and the continuous Galerkin (CG) method in the space variables. A similar approach was done for the first time using parabolic problems more specifically the diffusion equations in the nonsmooth domain as seen in [11]. The NSFD method was initiated by Mickens in [12] and major contributions to the foundation of the NSFD method could be seen in $[13,14]$. Since its initiation, the NSFD method has been extensively applied to many concrete problems in engineering and science; see $[12,15,16]$ for an overview. This paper compliments the technique used in [11]. The technique is geared toward obtaining a sufficiently smooth solution, the maximal error in the $L^{2}$-norm, and to show that the error across the entire interval convergences optimally as $\mathrm{O}\left(h^{2}+\right.$ $\left.\Delta t^{2}\right)$ where $h$ is the mesh size and $\Delta t$ is the time step size. The reliability of this technique comes from the fact that the NSFD-CG method preserves both the energy features and the hyperbolicity of the exact solution of the wave equation (1)(4).

The organization of the paper is as follows. Under Section 2, we review some of the useful spaces and their notations needed in the paper. In Section 3, we gather essential tools necessary to prove the main result of the paper. We present, in Section 4, a reliable scheme NSFD-CG and show that the numerical solution obtained from this scheme attains the optimal convergence rate in the energy as well as in the $L^{2}$-norms. Furthermore, we show that the scheme under consideration replicates the properties of the exact solution. Section 5 is devoted to some numerical experiments using a numerical example which confirms the optimal rate of convergence of the solution proved analytically in Section 4 . The concluding remarks are given in Section 6 and these underline how the work fits in the existing literature and also how it can be extended for further work.

\section{Notations}

In this section, we will review some of the spaces which we will be using in the paper together with their notations and possibly properties. For $s \geq 0, H^{s}(\Omega)$ will denote the Sobolev space of real-valued functions on $\Omega$, and the norm on $H^{s}(\Omega)$ will be denoted by $\|\cdot\|_{s}$. See [17] for the definitions and the relevant properties of these spaces. In a particular case, where $s=0$ the space $H^{0}(\Omega)=L^{2}(\Omega)$ and its inner product together with the norm will be denoted, respectively, by

$$
\begin{gathered}
(u, v)=\int_{\Omega} u v d x, \quad u, v \in L^{2}(\Omega), \\
\|u\|_{L^{2}(\Omega)}=\{(u, u)\}^{1 / 2}, \quad u \in L^{2}(\Omega) .
\end{gathered}
$$

In addition, $C_{0}^{\infty}(\Omega)$ will denote the space of infinitely differentiable functions with support compactly contained in $\Omega$. The space $H_{0}^{1}(\Omega)$ will denote the subspace of $H^{1}(\Omega)$ obtained by completing $C_{0}^{\infty}(\Omega)$ with respect to the norm $\|\cdot\|_{1}$. Following [17], for $X$ a Hilbert space, we will more generally use the Sobolev space $H^{s}[(0, T) ; X]$, where $s \geq 0$ and in the case where $s=0$ we will have $H^{0}[(0, T) ; X] \equiv$ $L^{2}[(0, T) ; X]$ with norm

$$
\|v\|_{L^{2}[(0, T) ; X]}=\left(\int_{0}^{T}\|v(\cdot, t)\|_{X} d t\right)^{1 / 2}
$$

In practice, $X$ will be the Sobolev space $H^{m}(\Omega)$ or $H_{0}^{m}(\Omega)$. Associated with (1) is the bilinear form

$$
a(u, v)=\int_{\Omega} \nabla u \nabla v d x, \quad u, v \in H^{1}(\Omega) .
$$

$a(\cdot, \cdot)$ will be symmetric and positive definite; that is,

$$
a(u, v)=a(v, u), \quad a(u, u) \geq 0
$$

\section{The Continuous Galerkin Method}

Having the previously mentioned notations in place, we proceed under this section to gather essential tools necessary to prove the main result of our paper. We begin by stating the following weak problem of (1)-(4).

Find $u \in L^{2}\left[(0, T) ; H_{0}^{1}(\Omega)\right]$ given $f \in L^{2}\left[(0, T) ; L^{2}(\Omega)\right]$ such that

$$
\begin{gathered}
\left(\frac{\partial^{2} u(\cdot, t)}{\partial t^{2}}, v\right)-a(u(\cdot, t), v)=(f(\cdot, t), v) \\
\forall v \in H_{0}^{1}(\Omega), t \geq 0, \\
(u(\cdot, 0), v)=\left(u_{0}, v\right), \\
\left(\frac{\partial u(\cdot, 0)}{\partial t}, v\right)=\left(u_{1}, v\right) .
\end{gathered}
$$

See [8] for the existence and the uniqueness of a solution $u$ of (9). Hence forth, in appropriate places to follow, additional conditions on the regularity of $u$ which guarantee the convergence results will be imposed. We continue next by providing the framework for stating the discrete version of (9). To this end, we let $\mathscr{T}_{h}$ be a regular family of triangulations of $\bar{\Omega}$ consisting of compatible triangles $T$ of diameter $h_{T} \leq$ $h$; see [17] for more. For each mesh size $\mathscr{T}_{h}$, we associate the finite element space $V_{h}$ of continuous piecewise linear function that are zero on the boundary

$$
V_{h}:=\left\{v_{h} \in C^{0}(\bar{\Omega}) ;\left.v_{h}\right|_{\partial \Omega}=0,\left.v_{h}\right|_{T} \in P_{1}, \forall T \in \mathscr{T}_{h}\right\}
$$

where $P_{1}$ is the space of polynomials of degree less than or equal to 1 and $V_{h}$ is a finite dimensional subspace of the Sobolev space $H_{0}^{1}(\Omega)$. It is well known that $V_{h}$ parametrized by $h \in(0,1)$ possesses the following approximation properties: there exists a constant $C$ such that if $v \in H_{0}^{1}(\Omega) \cap H^{2}(\Omega)$ we have

$$
\inf _{\chi \in V_{h}}\left\{\|v-\chi\|+h\|v-\chi\|_{1}\right\} \leq C h^{2}\|v\|_{2}
$$


By the use of the energy method and Gronwall's Lemma, there exists a discrete Galerkin solution $u_{h} \in V_{h}$ such that

$$
\begin{gathered}
\left(\frac{\partial^{2} u_{h}}{\partial t^{2}}, v_{h}\right)-a\left(u_{h}, v_{h}\right)=\left(f, v_{h}\right), \quad \forall v_{h} \in V_{h}, t \in[0, T], \\
\left(u_{h}, v_{h}\right)=\left(P_{h} u_{0}, v_{h}\right), \\
\left(\frac{\partial u_{h}}{\partial t}, v_{h}\right)=\left(P_{h} u_{1}, v_{h}\right),
\end{gathered}
$$

where $P_{h}$ denote the $L^{2}$-projection onto $V_{h}$. Furthermore, we let $u \in H^{2}(\Omega)$ and we define the Galerkin projection $\pi_{h} u$ of $u$ in $V_{h}$ by requiring that

$$
\begin{gathered}
a\left(\pi_{h} u, v\right)=a(u, v), \quad \forall v \in V_{h}, \\
\frac{\partial^{k}\left(\pi_{h} u\right)}{\partial t^{k}}=\pi_{h}\left(\frac{\partial^{k} u}{\partial t^{k}}\right), \quad k=0,1,2, t \in[0, T] .
\end{gathered}
$$

The previous essential tools lead to the immediate consequence of the approximation properties (11).

Lemma 1. Let $u$ be the solution of (9). Then, there exists a unique mapping $\pi_{h} u \in L^{2}\left[(0, T) ; V_{h}\right]$ which satisfies (13). Furthermore, if for some integer $k \geq 0, \partial^{k} u / \partial t^{k} \in$ $L^{p}\left[(0, T) ; H^{2}(\Omega)\right]$, then

$$
\begin{gathered}
\frac{\partial^{k}\left(\pi_{h} u\right)}{\partial t^{k}} \in L^{p}\left[(0, T) ; V_{h}\right] \\
\left\|\left(\frac{\partial}{\partial t}\right)^{k}\left[u-\pi_{h} u\right]\right\|_{L^{p}\left[(0, T) ; L^{2}(\Omega)\right]} \leq C h^{2}\left\|\left(\frac{\partial}{\partial t}\right)^{k} u\right\|_{L^{p}\left[(0, T) ; H^{2}(\Omega)\right]}
\end{gathered}
$$

for some constant $C$ independent of the mesh size.

\section{Coupled Nonstandard Finite Difference and Continuous Galerkin Methods}

Instead of the continuous Galerkin method summarized previously, we present in this section a reliable scheme NSFDCG consisting of the nonstandard finite difference method in time and the continuous Galerkin method in the space variable. We show that the numerical solution obtained from the scheme NSFD-CG attained the optimal convergence in both the energy and $L^{2}$-norms. We proceed, in this regard, by letting the step size $t_{n}=n \Delta t$ for $n=0,1,2, \ldots, N$. For a sufficiently smooth function $v(x, t)$, we set

$$
\left(\frac{\partial}{\partial t}\right)^{k} v^{n}=\left(\frac{\partial}{\partial t}\right)^{k} v\left(\cdot, t_{n}\right), \quad v^{n}=v\left(\cdot, t_{n}\right), k \geq 0 .
$$

With this, we proceed to find the NSFD-CG approximation $\left\{U_{h}^{n}\right\}$ such that $U_{h}^{n} \approx u_{h}^{n}$ at discrete time $t_{n}$. That is, find a sequence $\left\{U_{h}^{n}\right\}_{n=0}^{N}$ in $V_{h}$ such that

$$
\begin{gathered}
\left(\delta^{2} U_{h}^{n}, v_{h}\right)+a\left(U_{h}^{n}, v_{h}\right)=\left(f^{n}, v_{h}\right), \\
\forall v_{h} \in V_{h}, n=1,2, \ldots, N-1,
\end{gathered}
$$

where

$$
\delta^{2} U_{h}^{n}=\frac{U_{h}^{n+1}-2 U_{h}^{n}+U_{h}^{n-1}}{(\psi(\Delta t))^{2}}, \quad n=1,2, \ldots, N-1
$$

and $\psi(\Delta t)=2 \sin (\Delta t / 2)$ restricted between $0<\psi(\Delta t)<1$. The initial conditions $U_{h}^{0} \in V_{h}$ and $U_{h}^{1} \in V_{h}$ are given by

$$
\begin{gathered}
\left(U_{h}^{0}, v_{h}\right)=\left(P_{h} u_{0}, v_{h}\right), \\
\left(U_{h}^{1}, v_{h}\right)=\left(U_{h}^{0}+\psi(\Delta t) P_{h} u_{1}+\frac{(\psi(\Delta t))^{2}}{2} \widetilde{U}_{h}^{0}, v_{h}\right), \\
\forall v_{h} \in V_{h},
\end{gathered}
$$

where $\widetilde{U}_{h}^{0} \in V_{h}$ is defined by

$$
\left(\widetilde{U}_{h}^{0}, v_{h}\right)=\left(f^{0}, v_{h}\right)-a\left(u_{0}, v_{h}\right), \quad \forall v_{h} \in V_{h} .
$$

If $f=0$ in (1), we will have in view of (17) an exact scheme

$$
\left(\frac{U_{h}^{n+1}-2 U_{h}^{n}+U_{h}^{n-1}}{4 \sin ^{2}(\Delta t / 2)}, v_{h}\right)+\left(\nabla U_{h}^{n}, \nabla v_{h}\right)=0,
$$

which according to Mickens [12] replicates both the energy preserving features and the properties of the exact solution (1)-(4). In order to state and prove the main result, we need a framework on which this result is based. To this end, we proceed by decomposing the error denoted by $e^{n}=u^{n}-U_{h}^{n}$ into the following error equation:

$$
e^{n}=u^{n}-w_{h}^{n}+w_{h}^{n}-U_{h}^{n}=\eta^{n}-\phi^{n},
$$

where $w_{h}^{n}=\pi_{h} u^{n} \in V_{h}$ is the Galerkin projector of $u^{n}$. Due to the regularity assumption mentioned earlier, the exact solution $u$ of (1)-(4) satisfies

$$
\begin{array}{r}
\left(\frac{\partial^{2} u^{n}}{\partial t^{2}}, v_{h}\right)+a\left(u^{n}, v_{h}\right)=\left(f^{n}, v_{h}\right), \\
\forall v_{h} \in V_{h}, n=1,2, \ldots, N .
\end{array}
$$

Subtracting (17) from (24) and using some properties of the Galerkin projection in the space we have

$$
\begin{gathered}
\left(\delta^{2} w_{h}^{n}-\delta^{2} U_{h}^{n}, v_{h}\right)+a\left(w_{h}^{n}-u^{n}, v_{h}\right) \\
=\left(\delta^{2} w_{h}^{n}-\frac{\partial^{2} u^{n}}{\partial t^{2}}, v_{h}\right),
\end{gathered}
$$

from where we obtain in view of (23)

$$
\begin{gathered}
\left(\delta^{2} \phi^{n}, v_{h}\right)+\left(\phi^{n}, v_{h}\right)=\left(r^{n}, v_{h}\right), \\
\forall v_{h} \in V_{h}, n=1,2, \ldots, N-1,
\end{gathered}
$$

where $r^{n}=\delta^{2} w_{h}^{n}-\partial^{2} u^{n} / \partial t^{2}$. In view of the necessity for error bound, we set

$$
r^{n}= \begin{cases}\delta^{2} w_{h}^{n}-\frac{\partial^{2} u^{n}}{\partial t^{2}}, & \text { for } n=1,2, \ldots, N-1 \\ \frac{\phi^{1}-\phi^{0}}{(\psi(\Delta t))^{2}} & \text { for } n=0,\end{cases}
$$


from where we define

$$
R^{n}=\psi(\Delta t) \sum_{m=0}^{n} r^{m}
$$

The previously mentioned framework leads to the following main result.

Theorem 2. Let $u$ be the solution of (9) and $\left\{U_{h}^{n}\right\}_{n=0}^{N}$ in $V_{h}$ a sequence defined by (17)-(21). Suppose that $u \in$ $L^{2}\left[(0, T) ; H^{2}(\Omega)\right], \partial u / \partial t \in L^{2}\left[(0, T) ; H^{2}(\Omega)\right]$, and $\partial^{k} u / \partial t^{k} \in$ $L^{2}\left[(0, T) ; L^{2}(\Omega)\right]$ for $k=3,4$. Then, there exists a constant $C>0$ independent of $\Delta t$ and the mesh refinement size $h$ :

$$
\max _{0 \leq n \leq N}\left\|u(\cdot, \Delta t)-U_{h}^{n}\right\|_{0} \leq C\left[h^{2}+(\Delta t)^{2}\right]
$$

Furthermore, the discrete solution replicates all the properties of solution of the hyperbolic equation in the limiting case of the space independent equation.

We prove the previous Theorem 2 thanks to the following series of results.

Proposition 3. The following result holds for a constant $C>0$ that is independent of $\Delta t$ and the mesh refinement size $h$ :

$$
\begin{aligned}
\max _{n=0, \ldots, N}\left\|e^{n}\right\|_{0} \leq C( & \left\|e^{0}\right\|_{0}+\max _{n=0, \ldots, N}\left\|\eta^{n}\right\|_{0} \\
& \left.+\psi(\Delta t) \max _{n=0, \ldots, N-1}\left\|R^{n}\right\|_{0}\right) .
\end{aligned}
$$

Proof. In view of (23), we have the following relation using triangular inequality:

$$
\max _{n=0, \ldots, N}\left\|e^{n}\right\|_{0} \leq \max _{n=0, \ldots, N}\left\|\phi^{n}\right\|_{0}+\max _{n=0, \ldots, N}\left\|\eta^{n}\right\|_{0} .
$$

We bound $\phi^{n}$ in (31) by first taking partial sums of the first term of (26), seconded by adding the remaining terms from $n=1$ to $m$ for $1 \leq m \leq N-1$, and multiplying both sides by $\psi(\Delta t)$ yields

$$
\begin{aligned}
& \left(\frac{\phi^{m+1}-\phi^{m}}{\psi(\Delta t)}, v_{h}\right)-\left(\frac{\phi^{1}-\phi^{0}}{\psi(\Delta t)}, v_{h}\right)+\psi(\Delta t) \sum_{n=1}^{m} a\left(\phi^{m}, v_{h}\right) \\
& =\psi(\Delta t) \sum_{n=1}^{m}\left(r^{n}, v_{h}\right) .
\end{aligned}
$$

In view of (28), we can define

$$
\begin{gathered}
\Phi^{m}=\psi(\Delta t) \sum_{n=1}^{m} \phi^{m}, \\
\Phi^{0}=0
\end{gathered}
$$

and using this in (32) we have

$$
\left(\frac{\phi^{m+1}-\phi^{m}}{\psi(\Delta t)}, v_{h}\right)+a\left(\Phi^{m}, v_{h}\right)=\left(R^{m}, v_{h}\right), \quad \forall v_{h} \in V_{h} .
$$

If we choose $v_{h}=\phi^{m+1}+\phi^{m} \in V_{h}$ in (34) and multiply the result by $\psi(\Delta t)$, this yields

$$
\begin{gathered}
\left\|\phi^{m+1}\right\|_{0}^{2}-\left\|\phi^{m}\right\|_{0}^{2}+\psi(\Delta t) a\left(\Phi^{m}, \phi^{m+1}+\phi^{m}\right) \\
=\psi(\Delta t) \sum_{m=1}^{n-1}\left(R^{m}, \phi^{m+1}+\phi^{m}\right), \\
\text { for } 0 \leq m \leq N-1 .
\end{gathered}
$$

Summing this from $m=0$ to $m=n-1$ for $1 \leq n \leq N-1$ gives

$$
\begin{gathered}
\left\|\phi^{n}\right\|_{0}^{2}-\left\|\phi^{0}\right\|_{0}^{2}+\psi(\Delta t) \sum_{m=0}^{n-1} a\left(\Phi^{m}, \phi^{m+1}+\phi^{m}\right) \\
=\psi(\Delta t) \sum_{m=0}^{n-1}\left(R^{m}, \phi^{m+1}+\phi^{m}\right) .
\end{gathered}
$$

Since $a$ is symmetric, $\Phi^{0}=0$, and

$$
\Phi^{m+1}-\Phi^{m-1}=\psi(\Delta t)\left(\phi^{m}+\phi^{m+1}\right), \quad m=1,2, \ldots, N-1,
$$

then, we deduce in view of the third term of (36)

$$
\begin{aligned}
\psi(\Delta t) & \sum_{m=0}^{n-1} a\left(\Phi^{m}, \phi^{m+1}+\phi^{m}\right) \\
= & \sum_{m=0}^{n-1} a\left(\Phi^{m}, \Phi^{m+1}+\Phi^{m-1}\right) \\
= & \sum_{m=0}^{n-1} a\left(\Phi^{m}, \Phi^{m+1}\right) \\
& -\sum_{m=0}^{n-1} a\left(\Phi^{m+1}, \Phi^{m-1}\right) \\
= & a\left(\Phi^{n-1}, \Phi^{n}\right) .
\end{aligned}
$$

By symmetry and coercivity properties of $a$ and the fact that

$$
\Phi^{n}-\Phi^{n-1}=\psi(\Delta t) \phi^{n} \text { for } n=1,2, \ldots, N,
$$

we have in view of (38)

$$
\begin{aligned}
\frac{(\psi(\Delta t))^{2}}{2} a\left(\phi^{n}, \phi^{n}\right) \\
=\frac{1}{2} a\left(\Phi^{n}-\Phi^{n-i}, \Phi^{n}-\Phi^{n-1}\right) \\
=\frac{1}{2} a\left(\Phi^{n}, \Phi^{n}\right)-a\left(\Phi^{n}, \Phi^{n-1}\right) \\
\quad+\frac{1}{2} a\left(\Phi^{n-1}, \Phi^{n-1}\right)
\end{aligned}
$$


and so

$$
\frac{(\psi(\Delta t))^{2}}{2} a\left(\phi^{n}, \phi^{n}\right) \geq-a\left(\Phi^{n}, \Phi^{n-1}\right)
$$

and this together with (36) yields

$$
\begin{aligned}
& \left\|\phi^{n}\right\|_{0}^{2}-\frac{(\psi(\Delta t))^{2}}{2} a\left(\phi^{n}, \phi^{n}\right) \\
& \leq\left\|\phi^{0}\right\|_{0}^{2}+\psi(\Delta t) \sum_{m=0}^{n-1}\left(R^{n}, \phi^{m}+\phi^{m+1}\right), \\
& \quad \text { for } 1 \leq n \leq N .
\end{aligned}
$$

By Poincare inequality together with the continuity and coercivity of $a$, we have the following inequality:

$$
\begin{aligned}
\left\|\phi^{n}\right\|_{0}^{2} & -\frac{(\psi(\Delta t))^{2}}{2}\left\|\phi^{n}\right\|_{0}^{2} \\
& \leq\left\|\phi^{0}\right\|_{0}^{2}+\psi(\Delta t) \sum_{m=0}^{n-1}\left(R^{n}, \phi^{m}+\phi^{m+1}\right) .
\end{aligned}
$$

We suppose at this stage that $h$ and $\Delta t$ satisfy the CFL condition such that $\psi(\Delta t) / h<1$. With this condition, the previous inequality becomes

$$
\begin{array}{r}
C_{\Delta t}\left\|\phi^{n}\right\|_{0}^{2} \leq\left\|\phi^{0}\right\|_{0}^{2}+\psi(\Delta t) \sum_{m=0}^{n-1}\left(R^{n}, \phi^{m}+\phi^{m+1}\right), \\
1 \leq n \leq N
\end{array}
$$

from where we have $1 / 2<C_{\Delta t}=1-(\psi(\Delta t))^{2} / 2 h^{2}<1$ and $1 / 2<C_{\Delta t}<1$. By the use of Cauchy-Schwarz inequality on (44) and some algebraic manipulations, we have

$$
\begin{aligned}
C_{\Delta t}\left\|\phi^{n}\right\|_{0}^{2} \leq & \left\|\phi^{0}\right\|_{0}^{2}+\psi(\Delta t) \sum_{m=0}^{n-1}\left(R^{m}, \phi^{m}+\phi^{m+1}\right) \\
\leq & \left\|\phi^{0}\right\|_{0}^{2}+\psi(\Delta t) \sum_{m=0}^{n-1}\left\|R^{m}\right\|_{0}\left(\left\|\phi^{m}\right\|_{0}+\left\|\phi^{m+1}\right\|_{0}\right) \\
\leq & \left\|\phi^{0}\right\|_{0}^{2}+2 \psi(\Delta t) \sum_{m=0}^{n-1}\left\|R^{m}\right\|_{0_{m=0, \ldots, n}} \operatorname{mox}^{m} \|_{0} \\
\leq & \left\|\phi^{0}\right\|_{0}^{2}+\frac{C_{\Delta t}}{2} \max _{m=1, \ldots, n}\left\|\phi^{m}\right\|_{0}^{2} \\
& +\frac{2}{C_{\Delta t}}\left(\psi(\Delta t) \sum_{m=0}^{n-1}\left\|R^{n}\right\|_{0}\right)^{2} \text { for } 0 \leq m \leq n
\end{aligned}
$$

and since the right-hand side is independent of $n$, then

$$
\left(C_{\Delta t}-\frac{C_{\Delta t}}{2}\right)\left\|\phi^{m}\right\|_{0}^{2} \leq\left\|\phi^{0}\right\|_{0}^{2}+\frac{2}{C_{\Delta t}}\left(\psi(\Delta t) \sum_{n=0}^{N-1}\left\|R^{n}\right\|_{0}\right)^{2}
$$

which then implies that

$$
\frac{C_{\Delta t}}{2}\left\|\phi^{m}\right\|_{0}^{2} \leq\left\|\phi^{0}\right\|_{0}^{2}+\frac{2}{C_{\Delta t}}\left(\psi(\Delta t) \sum_{n=0}^{N-1}\left\|R^{n}\right\|_{0}\right)^{2} .
$$

Furthermore, we have

$$
\left\|\phi^{n}\right\|_{0}^{2} \leq \frac{2}{C_{\Delta t}}\left\|\phi^{n}\right\|_{0}^{2}+\frac{4(\psi(\Delta t))^{2}}{C_{\Delta t}}\left(\sum_{n=0}^{N-1}\left\|R^{n}\right\|_{0}\right)^{2}
$$

and hence the following result:

$$
\max _{n=0, \ldots, N}\left\|\phi^{n}\right\|_{0} \leq \sqrt{\frac{2}{C_{\Delta t}}}\left\|\phi^{0}\right\|_{0}+\frac{2 \psi(\Delta t)}{C_{\Delta t}} \sum_{n=1}^{N-1}\left\|R^{n}\right\|_{0} .
$$

With (49) and the fact that

$$
\left\|\phi^{0}\right\|_{0} \leq\left\|e^{0}\right\|_{0}+\left\|\eta^{0}\right\|_{0}
$$

we have in view of (31) the desired estimate of the proposition.

We now proceed to bound the term containing $R^{n}$ on the right-hand side of Proposition 3. We achieve this by estimating in the $L^{2}$-norm the function $r^{n}$ for the cases $n=0$ and follow by the case when $n \geq 1$.

Lemma 4. There holds

$$
\begin{array}{r}
\left\|r^{0}\right\|_{0} \leq C\left[(\psi(\Delta t))^{-1} h^{2}\left\|\frac{\partial u}{\partial t}\right\|_{L^{2}\left[(0, T) ; H^{2}(\Omega)\right]}\right. \\
\left.+\psi(\Delta t)\left\|\frac{\partial^{2} u}{\partial t^{2}}\right\|_{L^{2}\left[(0, T) ; L^{2}(\Omega)\right]}\right],
\end{array}
$$

with a constant $C>0$ that is independent of $h$ and the mesh size.

Proof. In view of (27), we have $r^{0}=(\psi(\Delta t))^{-2}\left(\phi^{1}-\phi^{0}\right)$. We estimate $\left\|\phi^{1}-\phi^{0}\right\|_{0}$ by taking $v_{h} \in V_{h}$ arbitrary as follows:

$$
\begin{aligned}
\left(\phi^{1}-\right. & \left.\phi^{0}, v_{h}\right) \\
& =\left(w_{h}^{1}-U_{h}^{1}, v_{h}\right)-\left(w_{h}^{0}-U_{h}^{0}, v_{h}\right) \\
& =\left(\left(\pi_{h}-I\right)\left(u_{1}-u_{0}\right), v_{h}\right)+\left(u_{1}-U^{1}, v_{h}\right),
\end{aligned}
$$

where we have used $\left(u_{0}-U_{h}^{0}, v_{h}\right)=\left(u_{0}-P_{h} u_{0}, v\right)=0$ in view of (19). It now follows from (52) that

$$
\begin{aligned}
& \left|\left(\left(\pi_{h}-I\right)\left(u_{1}-u_{0}\right), v_{h}\right)\right| \\
& \quad \leq \int_{0}^{t_{1}}\left|\left(\frac{\partial}{\partial t}\left(\pi_{h}-I\right) u(\cdot, s), v_{h}\right)\right| d s \quad \text { By Lemma } 1 \\
& \quad \leq \int_{0}^{t_{1}}\left|\left(\left(\pi_{h}-I\right) \frac{\partial u(\cdot, s)}{\partial t}, v_{h}\right)\right| d s \quad \text { By }(18) \\
& \quad \leq C \psi(\Delta t) h^{2}\left\|\frac{\partial u}{\partial t}\right\|_{L^{2}\left[(0, T) ; H^{2}(\Omega)\right]}\left\|v_{h}\right\|_{0} .
\end{aligned}
$$


We now proceed to estimate the term $\left(u_{1}-U_{h}^{1}, v\right)$ in (52) as follows: by Taylor's formula and the fact $u(\cdot, 0)=u_{0}$ and $\partial u(\cdot, 0) / \partial t=u_{1}$ in (1)-(3), we have

$$
\begin{aligned}
u_{1}= & u_{0}+\psi(\Delta t) u_{1}+\frac{(\psi(\Delta t))^{2}}{2} \frac{\partial^{2} u(\cdot, 0)}{\partial t^{2}} \\
& +\frac{1}{2} \int_{0}^{t_{1}}(\psi(\Delta t)-s)^{2} \frac{\partial^{3} u(\cdot, s)}{\partial t^{3}} d s .
\end{aligned}
$$

In view of the definition of $U_{h}^{1}$ in (20) and the fact that

$$
\left(u_{0}-P_{h} u_{0}, v\right)=0, \quad\left(u_{1}-P_{h} u_{1}, v\right)=0
$$

we have

$$
U_{h}^{1}=U_{h}^{0}+\psi(\Delta t) P_{h} u_{1}+\frac{(\psi(\Delta))^{2}}{2} \widetilde{U}_{h}^{0} .
$$

We then deduce from (54) and (56) that

$$
\begin{aligned}
\left(u_{1}-U_{h}^{1}, v_{h}\right)= & \frac{(\psi(\Delta t))^{2}}{2}\left(\frac{\partial^{2} u(\cdot, 0)}{\partial t^{2}}-\widetilde{U}_{h}^{0}, v_{h}\right) \\
& +\frac{1}{2} \int_{0}^{t_{1}}(\psi(\Delta t)-s)^{2}\left(\frac{\partial^{3} u(\cdot, s)}{\partial t^{3}}, v_{h}\right) d s .
\end{aligned}
$$

But by the definition of $\widetilde{U}_{h}^{0}$ in (20) we have in view of (21) that

$$
\begin{aligned}
& \left(\frac{\partial^{2} u(\cdot, s)}{\partial t^{2}}-\widetilde{U}_{h}^{0}, v_{h}\right) \\
& =\left(f^{0}, v_{h}\right)-a\left(u_{0}, v_{h}\right)-\left(f^{0}, v_{h}\right)+a\left(u_{0}, v_{h}\right)=0
\end{aligned}
$$

implying that

$$
\begin{aligned}
& \left|\left(u_{1}-U_{h}^{1}, v\right)\right| \\
& \quad \leq \frac{1}{2} \int_{0}^{t_{1}}(\psi(\Delta t)-s)^{2}\left|\left(\frac{\partial^{3} u(\cdot, s)}{\partial t^{3}}, v\right)\right| d s \\
& \quad \leq C(\psi(\Delta t))^{3}\left\|\frac{\partial^{3} u}{\partial t^{3}}\right\|_{L^{2}\left[(0, T) ; L^{2}(\Omega)\right]}\|v\|_{0} .
\end{aligned}
$$

Since $\phi^{1}-\phi^{0} \in V_{h}$, then (52) together with (53) and (59) yields

$$
\begin{aligned}
\left\|\phi^{1}-\phi^{0}\right\|_{0} \leq C( & \psi(\Delta t) h^{2}\left\|\frac{\partial u}{\partial t}\right\|_{L^{2}\left[(0, T) ; H^{2}(\Omega)\right]} \\
& \left.+(\psi(\Delta t))^{3}\left\|\frac{\partial^{3} u}{\partial t^{3}}\right\|_{L^{2}\left[(0, T) ; L^{2}(\Omega)\right]}\right)
\end{aligned}
$$

and dividing throughout by $(\psi(\Delta t))^{2}$ yields

$$
\begin{gathered}
\left\|r^{0}\right\|_{0} \leq C\left((\psi(\Delta t))^{-1} h^{2}\left\|\frac{\partial u}{\partial t}\right\|_{L^{2}\left[(0, T) ; H^{2}(\Omega)\right]}\right. \\
\left.+\psi(\Delta t)\left\|\frac{\partial^{3} u}{\partial t^{3}}\right\|_{L^{2}\left[(0, T) ; L^{2}(\Omega)\right]}\right)
\end{gathered}
$$

as required.
Lemma 5. For $1 \leq n \leq N-1$, there holds

$$
\begin{aligned}
\left\|r^{n}\right\|_{0} \leq C\left(\frac{h^{2}}{\psi(\Delta t)} \int_{t_{n-1}}^{t_{n+1}}\left\|\frac{\partial^{2} u(\cdot, s)}{\partial t^{2}}\right\|_{2} d s\right. \\
\left.+\psi(\Delta t) \int_{t_{n-1}}^{t_{n+1}}\left\|\frac{\partial^{4} u(\cdot, s)}{\partial t^{4}}\right\|_{0} d s\right)
\end{aligned}
$$

with a constant $C>0$ that is independent of $h$ and mesh size.

Proof. In view of (27), we have

$$
r^{n}=\delta^{2} w_{h}^{n}-\frac{\partial^{2} u^{n}}{\partial t^{2}}, \quad \text { for } n=1,2, \ldots, N-1 .
$$

By the triangular inequality, we have

$$
\begin{aligned}
\left\|r^{n}\right\|_{0} & =\left\|\delta^{2} w_{h}^{n}-\frac{\partial^{2} u^{n}}{\partial t^{2}}\right\|_{0} \\
& \leq\left\|\delta^{2}\left(\pi_{h}-I\right) u^{n}\right\|_{0}+\left\|\delta^{2} w_{h}^{n}-\frac{\partial^{2} u^{n}}{\partial t^{2}}\right\|_{0}
\end{aligned}
$$

and using the following identity

$$
\begin{aligned}
& v\left(\cdot, t_{n+1}\right)-2 v\left(\cdot, t_{n}\right)+v\left(\cdot, t_{n-1}\right) \\
& =\Delta t \int_{t_{n-1}}^{t_{n+1}}\left(1-\frac{\left|s-t_{n}\right|}{\Delta t}\right) \frac{\partial^{2} v(\cdot, s)}{\partial t^{2}} d s
\end{aligned}
$$

on the first term of the right-hand side of (64) we have

$$
\begin{aligned}
& \left\|\delta^{2}\left(\pi_{h}-I\right) u^{n}\right\|_{0} \\
& \quad \leq \frac{1}{\psi(\Delta t)} \int_{t_{n-1}}^{t_{n+1}}\left(1-\frac{\left|s-t_{n}\right|}{\Delta t}\right)\left\|\frac{\partial^{2}\left(\pi_{h}-I\right) u}{\partial t^{2}}\right\|_{0} d s \\
& \quad \leq C \frac{h^{2}}{\psi(\Delta t)} \int_{t_{n-1}}^{t_{n+1}}\left\|\frac{\partial^{2} u(\cdot, s)}{\partial t^{2}}\right\|_{2} d s
\end{aligned}
$$

after the use of Lemma 1 and (14).

The second term of (64) can be estimated by the use of the identity

$$
\begin{aligned}
\delta^{2} w_{h}^{n} & -\frac{\partial^{2} u^{n}}{\partial t^{2}} \\
& =\frac{1}{6(\Delta t)^{2}} \int_{t_{n-1}}^{t_{n+1}}\left(\Delta t-\left|s-t_{n}\right|\right)^{3} \frac{\partial^{4} u(\cdot, s)}{\partial t^{4}} d s
\end{aligned}
$$

which is obtained from Taylor's formulae with integral remainder. This is deduced as follows:

$$
\begin{gathered}
\left\|\delta^{2} w_{h}^{n}-\frac{\partial^{2} u^{n}}{\partial t^{2}}\right\|_{0} \\
\leq \frac{1}{6(\Delta t)^{2}} \int_{t_{n-1}}^{t_{n+1}}\left(\Delta t-\left|s-t_{n}\right|\right)^{3}\left\|\frac{\partial^{4} u(\cdot, s)}{\partial t^{4}}\right\|_{0} d s \\
\leq \frac{1}{6(\Delta t)^{2}} \int_{t_{n-1}}^{t_{n+1}}(\psi(\Delta t))^{3}\left\|\frac{\partial^{4} u(\cdot, s)}{\partial t^{4}}\right\|_{0} d s \\
\leq \frac{\psi(\Delta t)}{6} \int_{t_{n-1}}^{t_{n+1}}\left\|\frac{\partial^{4} u(\cdot, s)}{\partial t^{4}}\right\|_{0} d s
\end{gathered}
$$

using the relation that $\psi(\Delta t)-\left|s-t_{n}\right| \leq \psi(\Delta t)$ in (68). 
In view of (64) using (66) and (68), we have the desired result for the bound of $\left\|r^{n}\right\|_{0}$. We now assemble Lemmas 4 and 5 in the next proposition to obtain the bound $R^{n}$ as follows.

Proposition 6. For $0 \leq n \leq N-1$, there holds

$$
\begin{aligned}
& \left\|R^{n}\right\|_{0} \\
& \leq \quad C(\psi(\Delta t))^{2}\left(\left\|\frac{\partial^{3} u}{\partial t^{3}}\right\|_{L^{2}\left[(0, T) ; L^{2}(\Omega)\right]}+\left\|\frac{\partial^{4} u}{\partial t^{4}}\right\|_{L^{2}\left[(0, T) ; L^{2}(\Omega)\right]}\right) \\
& \quad+C h^{2}\left(\left\|\frac{\partial u}{\partial t}\right\|_{L^{2}\left[(0, T) ; H^{2}(\Omega)\right]}+\left\|\frac{\partial^{2} u}{\partial t^{2}}\right\|_{L^{2}\left[(0, T) ; H^{2}(\Omega)\right]}\right) .
\end{aligned}
$$

Proof. Using the bounds on $\left\|r^{n}\right\|_{0}$ in Lemmas 4 and 5, we have

$$
\begin{aligned}
\left\|R^{n}\right\|_{0} \leq & \psi(\Delta t)\left\|r^{0}\right\|_{0}+\psi(\Delta t) \sum_{m=1}^{N-1} r^{m} \\
\leq & C(\psi(\Delta t))^{2}\left(\left\|\frac{\partial^{3} u}{\partial t^{3}}\right\|_{L^{2}\left[(0, T) ; L^{2}(\Omega)\right]}\right. \\
& \left.+\left\|\frac{\partial^{4} u}{\partial t^{4}}\right\|_{L^{2}\left[(0, T) ; L^{2}(\Omega)\right]}\right) \\
& +C h^{2}\left(\left\|\frac{\partial u}{\partial t}\right\|_{L^{2}\left[(0, T) ; H^{2}(\Omega)\right]}+\left\|\frac{\partial^{2} u}{\partial t^{2}}\right\|_{L^{2}\left[(0, T) ; H^{2}(\Omega)\right]}\right)
\end{aligned}
$$

and the proof is completed.

With all these results, we are now in the position to prove the main result as follows.

Proof of Theorem 2. Using Proposition 3 and the fact that

$$
\psi(\Delta t) \sum_{n=0}^{N-1}\left\|R^{n}\right\|_{0} \leq T \max _{n=0, \ldots, N}\left\|R^{n}\right\|_{0}
$$

we have

$$
\max _{n=0, \ldots, N}\left\|e^{n}\right\| \leq C\left(\left\|e^{0}\right\|_{0}+\max _{n=0, \ldots, N}\left\|\eta^{n}\right\|_{0}+T \max _{n=0, \ldots, N-1}\left\|R^{n}\right\|_{0}\right) .
$$

By the use of Lemma 1, we can bound the second term on the right-hand side as follows.

$$
\max _{n=0, \ldots, N}\left\|\eta^{n}\right\|_{0} \leq C h^{2}\|u\|_{L^{2}\left[(0, T) ; H^{2}(\Omega)\right]} .
$$

From the approximation property of the $L^{2}$-projection, we have

$$
\left\|e^{0}\right\|_{0} \leq C h^{2}\left\|u_{0}\right\|_{2} \leq C h^{2}\|u\|_{L^{2}\left[(0, T) ; H^{2}(\Omega)\right]} .
$$

Finally, by the bound of $\max _{n=0, \ldots, N-1}\left\|R^{n}\right\|_{0}$ obtained via Proposition 6, we have the result

$$
\begin{aligned}
\max _{n=0, \ldots, N}\left\|e^{n}\right\|_{0} \leq & C h^{2}\|u\|_{L^{2}\left[(0, T) ; H^{2}(\Omega)\right]} \\
& +C(\psi(\Delta t))^{2}\left(\left\|\frac{\partial^{3} u}{\partial t^{3}}\right\|_{L^{2}\left[(0, T) ; L^{2}(\Omega)\right]}\right. \\
& \left.+\left\|\frac{\partial^{4} u}{\partial t^{4}}\right\|_{L^{2}\left[(0, T) ; L^{2}(\Omega)\right]}\right) .
\end{aligned}
$$

In view of the relationship

$$
\psi(\Delta t)=2 \sin \left(\frac{\Delta t}{2}\right) \approx \Delta t+0\left[(\Delta t)^{2}\right]
$$

as proposed for such schemes in Mickens [12], we have the required estimate as $\Delta t \rightarrow 0$. Furthermore, using the fact that its uniform convergence results imply pointwise convergence for $x \in \Omega^{\prime}$ completes the proof.

\section{Numerical Experiments}

In this section, we present the numerical experiments on problem (1) using both the standard finite difference (SFD) and NSFD-CG methods. These experiments are performed in $\Omega=(0,1)^{2} \times(0, T)$ where $\Omega$ was discretized using regular meshes of sizes $h=1 / M$ in the space and $\Delta t=T / N$ in the time. The $M$ and $N$ in such a discretization denote the number of nodes and time respectively. The initial data was considered to be $u_{0}(x)=x_{1} x_{2}\left(1-x_{1}\right)\left(1-x_{2}\right)$ and $u_{1}(x)=0$ where these data were deduced from the exact solution

$$
u(x, t)=x_{1} x_{2}\left(1-x_{1}\right)\left(1-x_{2}\right) \cos (\pi t) .
$$

Using the previous exact solution we obtained the right-hand side $f$ of (1). In the computation, (1) together with (17)-(20) led to a system of equations

$$
\mathbf{A X}=\mathbf{b} .
$$

In solving for $\mathbf{X}$ in the above system, we took the following values of $M=10,15,20,25,50$, and 100 . For a fix $M=20$, $N=10$, and $T=1$, we had Figures 1-3 illustrating various solutions corresponding to their respective schemes.

Figure 1 shows the exact solution, Figure 2 the solution from the SFD-CG, and Figure 3 the solution from the NSFDCG schemes, followed by Tables 1 and 2 which demonstrate various optimal rates of convergence in both $H^{1}$ and $L^{2}$ norms of these schemes.

These optimal rates of convergence were calculated by using the formula

$$
\text { Rate }=\frac{\ln \left(e_{2} / e_{1}\right)}{\ln \left(h_{2} / h_{1}\right)},
$$

where $h_{1}$ and $h_{2}$ together with $e_{1}$ and $e_{2}$ are successive triangle diameters and errors, respectively. These results are selfexplanatory and we could conclude that the results as shown by these experiments exhibit the desired results as expected from our theoretical analysis. 


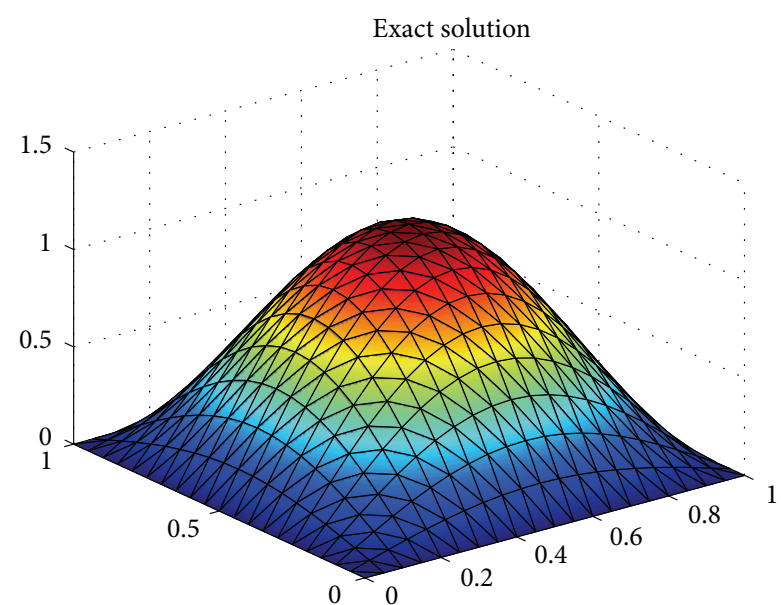

FIgURE 1: The Exact solution.

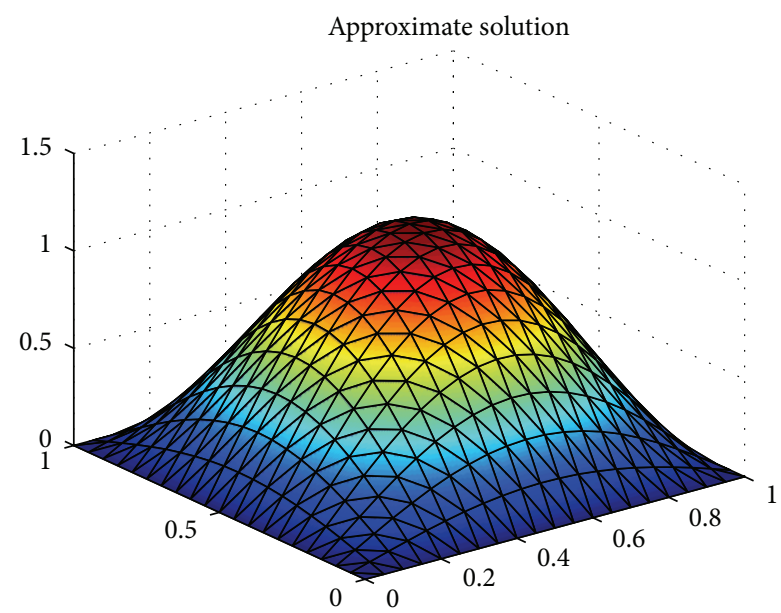

Figure 2: Approximate solution for SFD-CG scheme.

\section{Conclusion}

We presented a reliable scheme of the wave equation consisting of the nonstandard finite difference method in time and the continuous Galerkin method in the space variable (NSFD-CG). We proved theoretically that the numerical solution obtained from this scheme attains the optimal rate of convergence in both the energy and the $L^{2}$-norms. Furthermore, we showed that the scheme under investigation replicates the properties of the exact solution of the wave equation. We proceeded by the help of a numerical example and showed that the optimal rate of convergence as proved theoretically is guaranteed in both the energy and the $L^{2}$ norms. This convergence results hold for any fully discrete NSFD-CG method where the scheme under consideration has a bilinear form which is symmetric, continuous, and coercive.

The method presented in this paper can be extended to the nonlinear hyperbolic or parabolic problems with either smooth or nonsmooth domain if at all these cases followed the procedure as proposed by Mickens [12]. We will

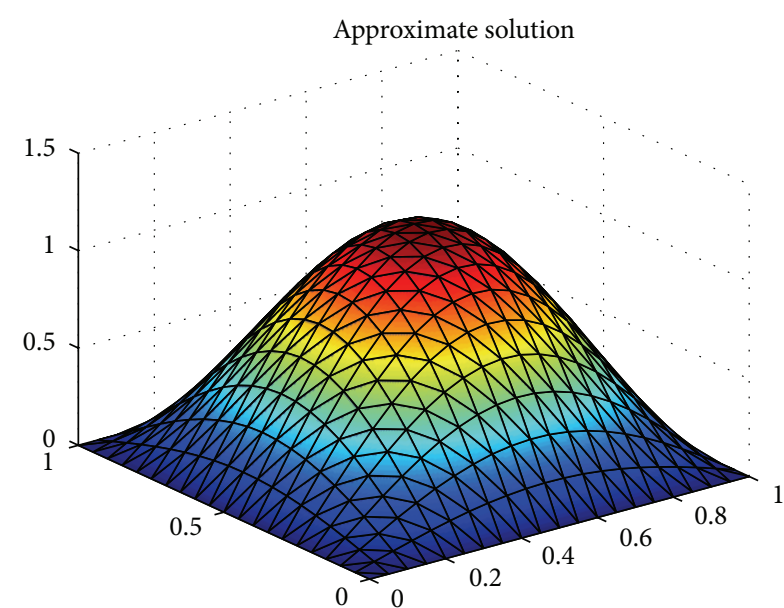

Figure 3: Approximate solution for NSFD-CG scheme.

TABLE 1: NSFD method error in both $L^{2}$ and $H^{1}$-norms.

\begin{tabular}{lcccc}
\hline$M$ & $L^{2}$-error & $H^{1}$-error & Rate $L^{2}$ & Rate $H^{1}$ \\
\hline 10 & $3.800 E-3$ & $1.67 E-2$ & & \\
15 & $1.700 E-3$ & $1.110 E-2$ & 1.983 & 1.0073 \\
20 & $1.000 E-3$ & $8.200 E-3$ & 1.8445 & 0.9688 \\
25 & $6.591 E-4$ & $6.700 E-3$ & 1.8678 & 1.0134 \\
50 & $2.012 E-4$ & $3.400 E-3$ & 1.7119 & 0.9786 \\
100 & $5.098 E-5$ & $1.800 E-3$ & 1.9806 & 0.9175 \\
\hline
\end{tabular}

TABLE 2: SFD method error in both $L^{2}$ and $H^{1}$-norms.

\begin{tabular}{lcccc}
\hline$M$ & $L^{2}$-error & $H^{1}$-error & Rate $L^{2}$ & Rate $H^{1}$ \\
\hline 10 & $3.400 E-3$ & $1.700 E-2$ & & \\
15 & $1.700 E-3$ & $1.130 E-2$ & 1.709 & 1.007 \\
20 & $1.050 E-3$ & $8.500 E-3$ & 1.674 & 0.989 \\
25 & $1.600 E-3$ & $6.800 E-3$ & 1.887 & 1.000 \\
50 & $5.170 E-4$ & $3.500 E-3$ & 1.629 & 0.958 \\
100 & $1.799 E-4$ & $1.900 E-3$ & 1.522 & 0.881 \\
\hline
\end{tabular}

also exploit another form of nonstandard finite differential method as proposed in $[18,19]$.

\section{Conflict of Interests}

The author declares that there is no conflict of interests regarding the publication of this paper.

\section{Acknowledgments}

Big thanks goes to the Almighty God from whose strength I draw my inspiration to complete this project. The research contained in this paper has been supported by the University of Pretoria, South Africa. The authors would like to thank the reviewers who spent a lot of time to go through the paper. Thanks also go to the following colleagues Dr. Chapwanya, Dr. Appadu, Mr. Mbehou, and Mr. Aderogba who help checked the revised version of the work. 


\section{References}

[1] D. A. French and J. W. Schaeffer, "Continuous finite element methods which preserve energy properties for nonlinear problems," Applied Mathematics and Computation, vol. 39, no. 3, pp. 271-295, 1990.

[2] D. A. French and T. E. Peterson, "A continuous space-time finite element method for the wave equation," Mathematics of Computation, vol. 65, no. 214, pp. 491-506, 1996.

[3] R. T. Glassey, "Convergence of an energy-preserving scheme for the Zakharov equations in one space dimension," Mathematics of Computation, vol. 58, no. 197, pp. 83-102, 1992.

[4] R. Glassey and J. Schaeffer, "Convergence of a second-order scheme for semilinear hyperbolic equations in $2+1$ dimensions," Mathematics of Computation, vol. 56, no. 193, pp. 87-106, 1991.

[5] W. Strauss and L. Vazquez, "Numerical solution of a nonlinear Klein-Gordon equation," Journal of Computational Physics, vol. 28, no. 2, pp. 271-278, 1978.

[6] T. Dupont, " $L^{2}$-estimates for Galerkin methods for second order hyperbolic equations," SIAM Journal on Numerical Analysis, vol. 10, pp. 880-889, 1973.

[7] G. A. Baker, "Error estimates for finite element methods for second order hyperbolic equations," SIAM Journal on Numerical Analysis, vol. 13, no. 4, pp. 564-576, 1976.

[8] E. Gekeler, "Linear multistep methods and Galerkin procedures for initial boundary value problems," SIAM Journal on Numerical Analysis, vol. 13, no. 4, pp. 536-548, 1976.

[9] C. Johnson, "Discontinuous Galerkin finite element methods for second order hyperbolic problems," Computer Methods in Applied Mechanics and Engineering, vol. 107, no. 1-2, pp. 117-129, 1993.

[10] G. R. Richter, "An explicit finite element method for the wave equation," Applied Numerical Mathematics, vol. 16, no. 1-2, pp. 65-80, 1994, A Festschrift to honor Professor Robert Vichnevetsky on his 65th birthday.

[11] P. W. M. Chin, J. K. Djoko, and J. M.-S. Lubuma, "Reliable numerical schemes for a linear diffusion equation on a nonsmooth domain," Applied Mathematics Letters, vol. 23, no. 5, pp. 544-548, 2010.

[12] R. E. Mickens, Nonstandard Finite Difference Models of Differential Equations, World Scientific Publishing, River Edge, NJ, USA, 1994.

[13] R. Anguelov and J. M.-S. Lubuma, "Contributions to the mathematics of the nonstandard finite difference method and applications," Numerical Methods for Partial Differential Equations, vol. 17, no. 5, pp. 518-543, 2001.

[14] R. Anguelov and J. M.-S. Lubuma, "Nonstandard finite difference method by nonlocal approximation," Mathematics and Computers in Simulation, vol. 61, no. 3-6, pp. 465-475, 2003.

[15] S. M. Moghadas, M. E. Alexander, B. D. Corbett, and A. B. Gumel, "A positivity-preserving Mickens-type discretization of an epidemic model," Journal of Difference Equations and Applications, vol. 9, no. 11, pp. 1037-1051, 2003.

[16] K. C. Patidar, "On the use of nonstandard finite difference methods," Journal of Difference Equations and Applications, vol. 11, no. 8, pp. 735-758, 2005.

[17] J. L. Lions, E. Magenes, and P. Kenneth, Non-Homogeneous Boundary Value Problems and Applications, vol. 1, Springer, Berlin, Germany, 1972.
[18] R. Uddin, "Comparison of the nodal integral method and nonstandard finite-difference schemes for the Fisher equation," SIAM Journal on Scientific Computing, vol. 22, no. 6, pp. 19261942, 2000.

[19] J. Oh and D. A. French, "Error analysis of a specialized numerical method for mathematical models from neuroscience," Applied Mathematics and Computation, vol. 172, no. 1, pp. 491507, 2006. 


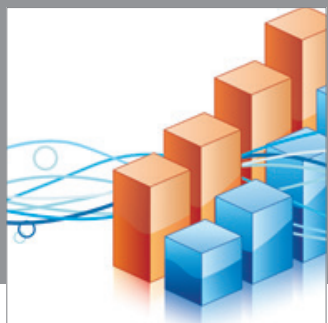

Advances in

Operations Research

mansans

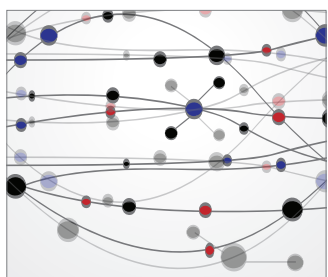

The Scientific World Journal
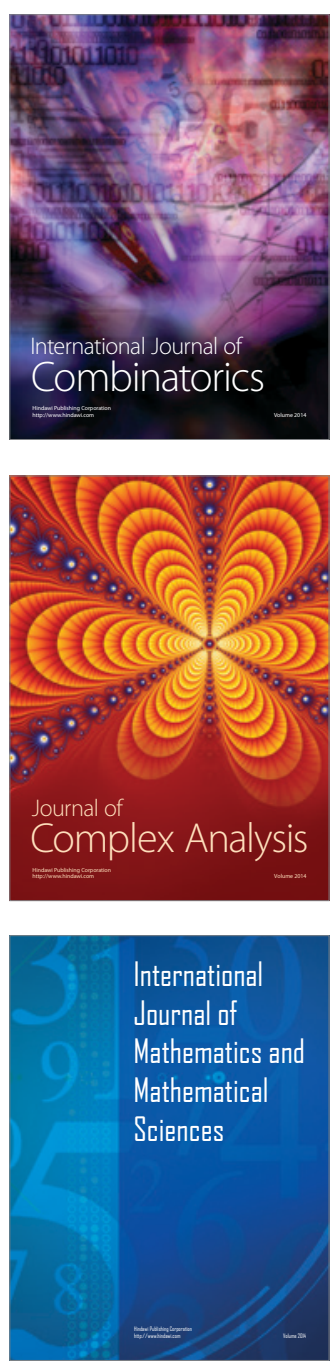
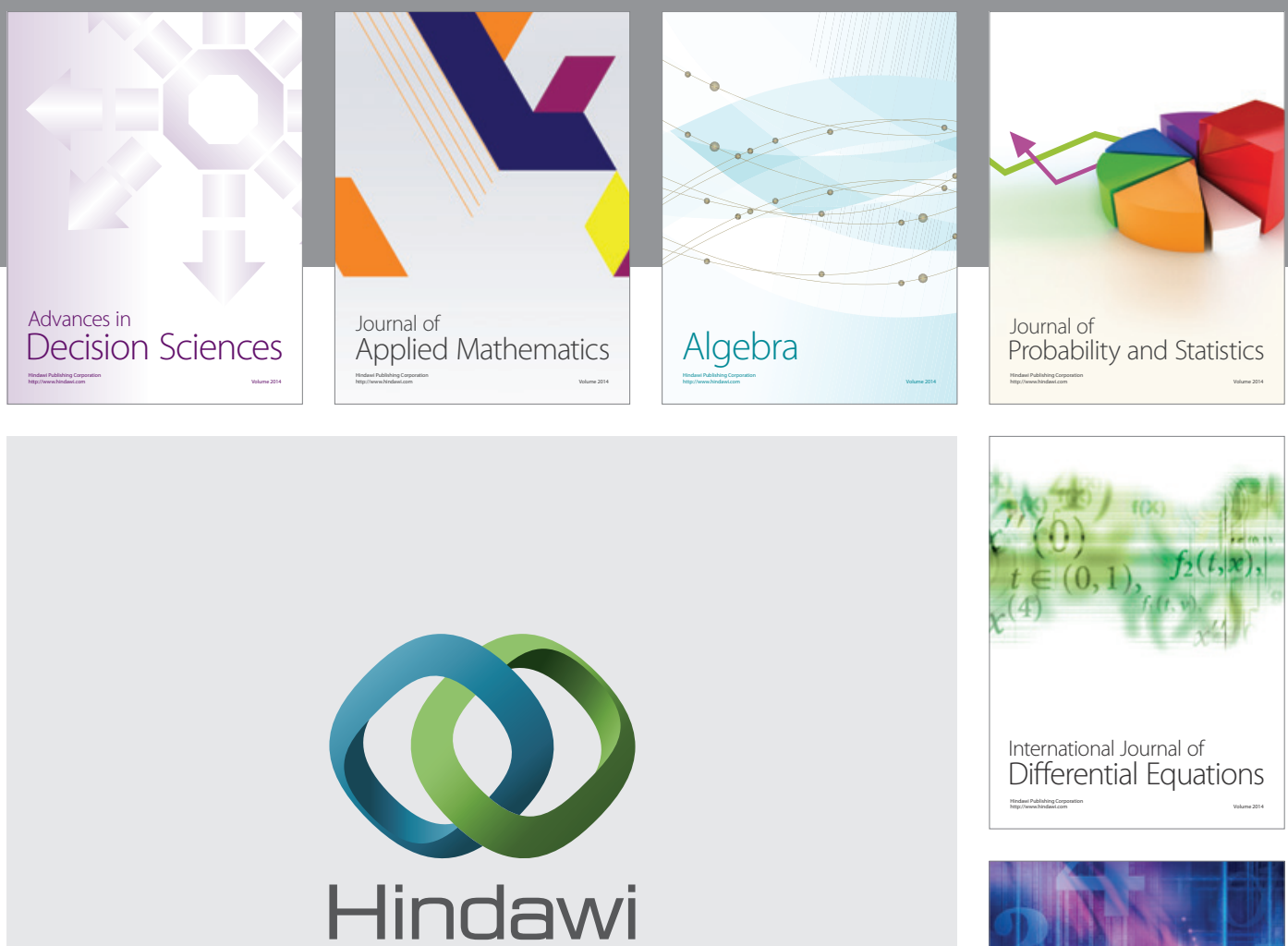

Submit your manuscripts at http://www.hindawi.com
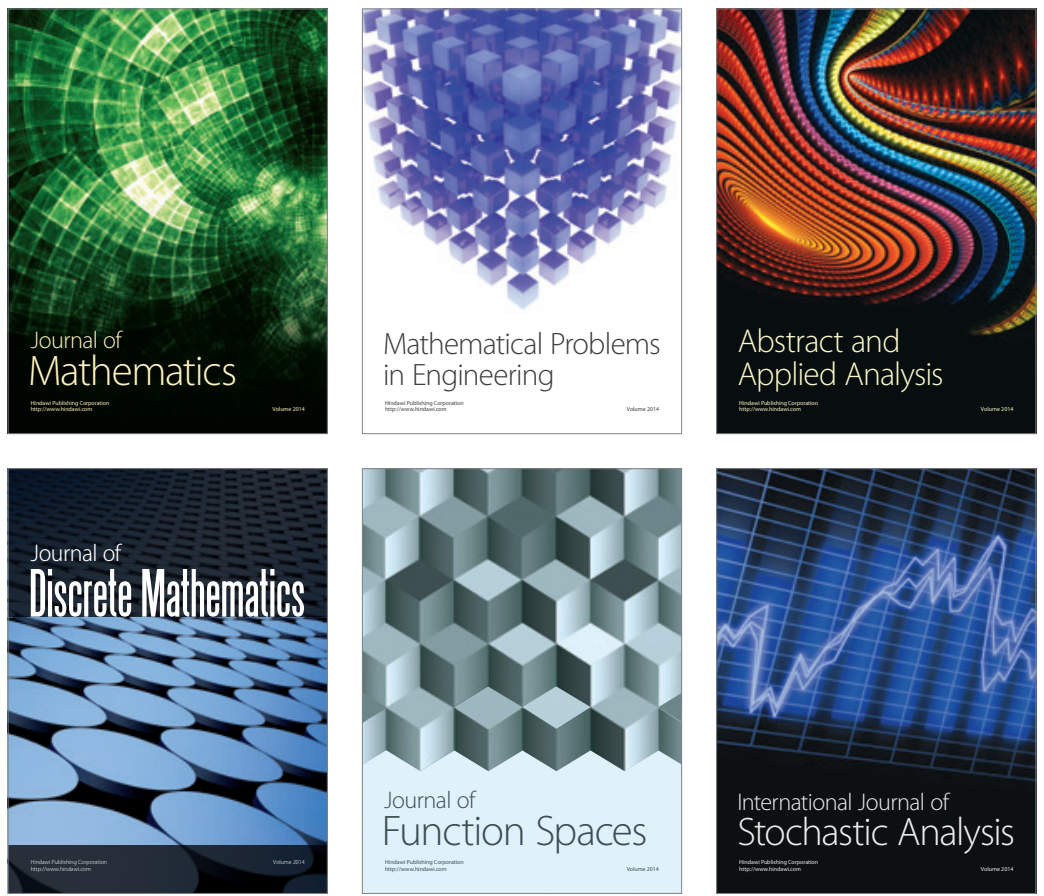

Journal of

Function Spaces

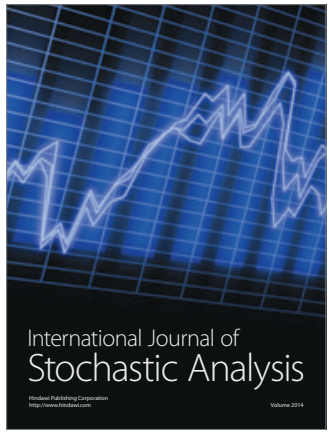

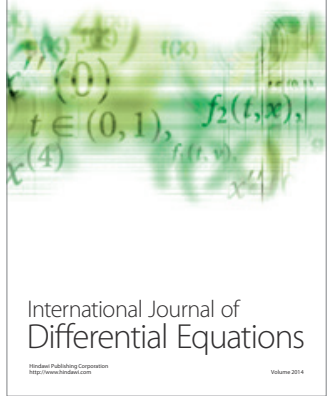
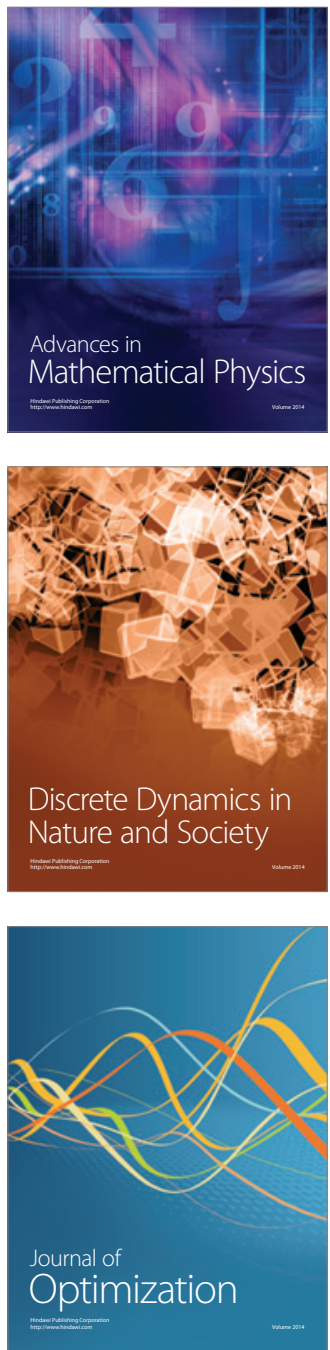